\title{
Biaxial Solar Tracking System Based on the MPPT Approach Integrating ICTs for Photovoltaic Applications
}

\author{
Raúl Gregor, ${ }^{1}$ Yoshihiko Takase, ${ }^{1}$ Jorge Rodas, ${ }^{1}$ Leonardo Carreras, ${ }^{1}$ \\ Derlis Gregor, ${ }^{2}$ and Andrés López ${ }^{1}$ \\ ${ }^{1}$ Department of Power and Control Systems, Faculty of Engineering, National University of Asunción, Campus Isla Bogado, \\ 2060 Luque, Paraguay \\ ${ }^{2}$ Department of Computer Science, Faculty of Engineering, National University of Asunción, Campus Isla Bogado, \\ 2060 Luque, Paraguay
}

Correspondence should be addressed to Raúl Gregor; rgregor@ing.una.py

Received 28 November 2014; Revised 19 March 2015; Accepted 24 March 2015

Academic Editor: Francisco A. S. Neves

Copyright (C) 2015 Raúl Gregor et al. This is an open access article distributed under the Creative Commons Attribution License, which permits unrestricted use, distribution, and reproduction in any medium, provided the original work is properly cited.

\begin{abstract}
The smart grid and distributed generation based on renewable energy applications often involve the use of information and communication technology (ICT) coupled with advanced control and monitoring algorithms to improve the efficiency and reliability of the electrical grid and renewable generation systems. Photovoltaic (PV) systems have been recently applied with success in the fields of distributed generation due to their lower environmental impact where the electrical energy generation is related to the amount of solar irradiation and thus the angle of incident ray of the sun on the surface of the modules. This paper introduces an integration of ICTs in order to achieve the maximum power point tracking (MPPT) using a biaxial solar tracking system for PV power applications. To generate the references for the digital control of azimuth and elevation angles a Global Positioning System (GPS) by satellites is used which enables acquiring the geographic coordinates of the sun in real-time. As a total integration of the system a communication platform based on the 802.15.4 protocol for the wireless sensor networks (WSNs) is adopted for supervising and monitoring the PV plant. A $2.4 \mathrm{~kW}$ prototype system is implemented to validate the proposed control scheme performance.
\end{abstract}

\section{Introduction}

Because of the growth global electricity demand projected by International Energy Agency (IEA) quantified around 81\% from 2011 to 2035 and the strong investment driven by an energy policy promoted by the Kyoto Protocol which has forced ratifying countries to reduce their emissions of greenhouse gases (GHG) in the recently approved second period from January 2013 to December 31, 2020. This scenario has been focused on the research effort in electricity generation from renewable energy (RE) sources. In this context, PV systems are currently considered as one of the most useful natural energy sources due to their continuous cost reduction of manufacturing, fast technological progress, requiring very little maintenance, and being pollution-free $[1,2]$. However, the power output of PV cells depends on many factors; these include the operating temperature, weather conditions, irradiance, and angle of incidence of the solar radiation [3]. In order to overcome these drawbacks, maximum power should be extracted from these systems using physical tracking to obtain the maximum power point tracking (MPPT). Taxonomy of the MPPT techniques applied to PV power system can be found in [4]. Physical tracking involves aligning the PV system, to be orthogonal to sun rays throughout the day in order to receive maximum solar radiation [5]. Several methods have been implemented and evaluated to keep the PV systems orthogonal to sun rays. An ideal solar tracker must ensure that the PV cell is oriented properly, compensating for both changes in the elevation angle of the sun (throughout the day) and latitudinal offset of the sun (during seasonal changes) and changes in azimuth angle. It has been shown in the literature that for certain geographical positions up to $40 \%$ extra power per year can be produced using a solar tracking system. These systems are usually 


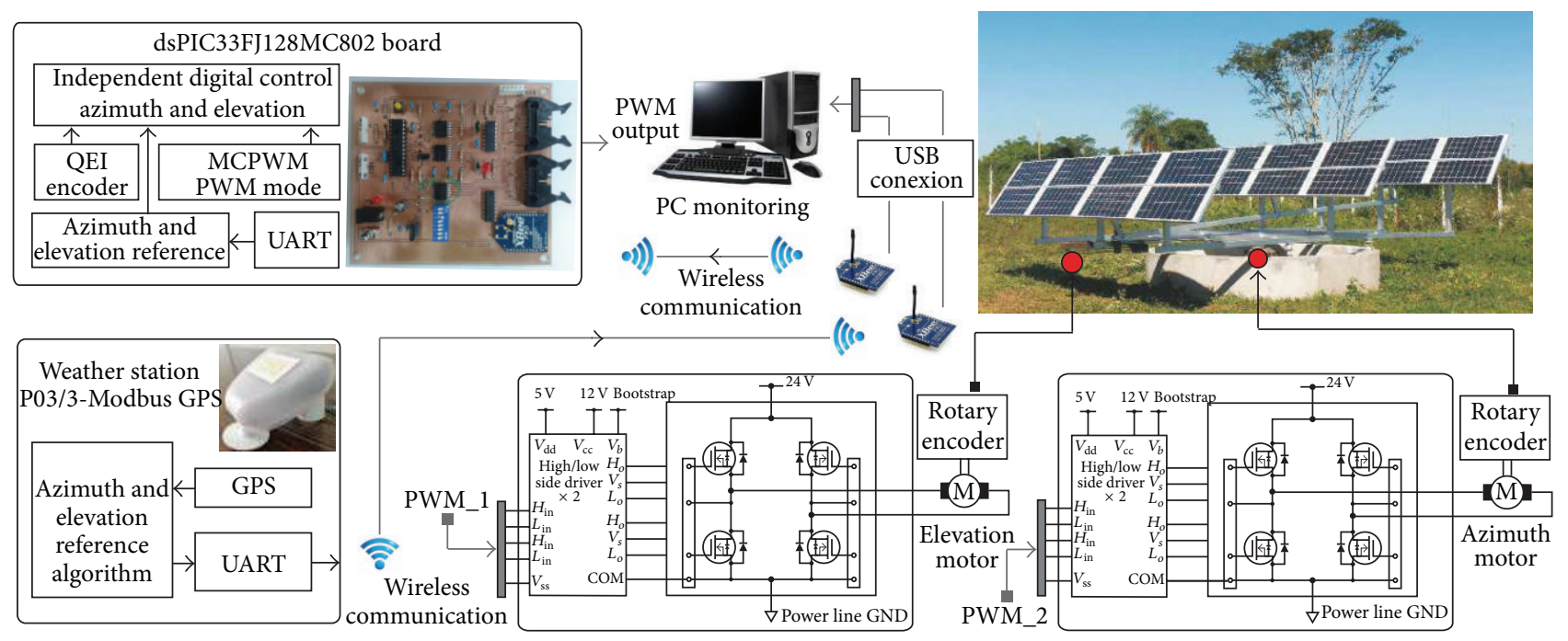

FIGURE 1: Block diagram of the proposed biaxial solar tracking system based on ICTs.

classified in two categories: passive, introduced by Finster in 1962 as a completely mechanical solution, and active that presented a mechanism with an automatic electronic control system. The above-mentioned classification of the methods can be found in [6]. Active trackers can be in turn classified by the type of electronic control which drives the movement: in the analog type the control is generated based on the information of a sensor that detects the position of the brightest point in the sky, while in the digital case, the control is executed by a microprocessor which through a control algorithm determines the optimal position of the structure to maximize the efficiency of the PV systems [7, 8]. In both cases, the sun position usually is detected by two lightdependent resistor (LDR) sensors that are normally located at both ends of the surface of the photovoltaic module. In the digital case, the resultant signals from the sensors are fed into an electronic control system that operates a low-speed DC motor to rotate the PV modules via a speed reduction system. This is a simple and inexpensive solution, but unfortunately it is not practical mainly in applications where weather conditions are variable or when fast change happens in the irradiance conditions due to partial shading $[9,10]$. This paper introduces a novel design of a biaxial solar digital tracking system for PV power applications, using information and communication technologies (ICTs) to improve the efficiency of the photovoltaic system. To generate the reference (in azimuth and elevation angle) for the digital control algorithm a Global Positioning System (GPS) by satellites is used which enables acquiring the geographic coordinates of the sun in real-time. The implementation of the control system is based on the dsPIC33FJ128MC802 microprocessor family manufactured by Microchip. For monitoring purposes the overall system also provides wireless sensor networks (WSNs) based on 802.15.4 protocol, as shown in the block diagram of Figure 1.

This paper is organized as follows. Section 2 introduces the diode and two-diode models for a PV module and parametric simulations for a ZDNY-100C36 series PV module.
Section 3 describes a custom-design process of the elevation and azimuth mechanism for the biaxial solar tracking system. Section 4 discusses the control strategy applied to the motors in order to achieve the MPPT. Next, experimental results are given in Section 5. The conclusion is given in the last section.

\section{PV Module Model Description}

Different approaches have been implemented to model mathematically the dynamics of the PV modules, but probably the equivalent circuit representation with single-diode and twodiode models, which are illustrated in Figure 2, represent the most widely used topologies [11-14]. The two-diode model, as shown in Figure 2(a), is characterized by its high accuracy; however, it is relatively complex and it suffers from low computational speed. The second type, single-diode model shown in Figure 2(b), is the most commonly used model in power electronic simulation studies, because it offers a reasonable tradeoff between simplicity and accuracy. Another advantage of using the single-diode model is the possibility to parametrize it based only on provided information by datasheet [15]. Because of this reason, this work focuses on the single-diode model. The simplest equivalent circuit of a PV cell is a current source in parallel with a diode as shown in Figure 2. The current source $\left(I_{\mathrm{ph}}\right)$ represents the current generated by the PV cell due to the photons received by it, and it has invariant value under constant solar irradiance and temperature. The equations that model the PV system performance may be obtained from circuit representation from Kirchoff's currents law, which results in the following equation:

$$
I_{\mathrm{ph}}-I_{D}-\frac{v_{d}}{R_{p}}-I_{\mathrm{pv}}=0,
$$

where $I_{D}$ and $I_{\mathrm{pv}}$ represent the diode and cell output currents, respectively, $v_{d}$ is the diode voltage, and $R_{p}$ is the shunt resistance in parallel with the diode. On the other hand, 


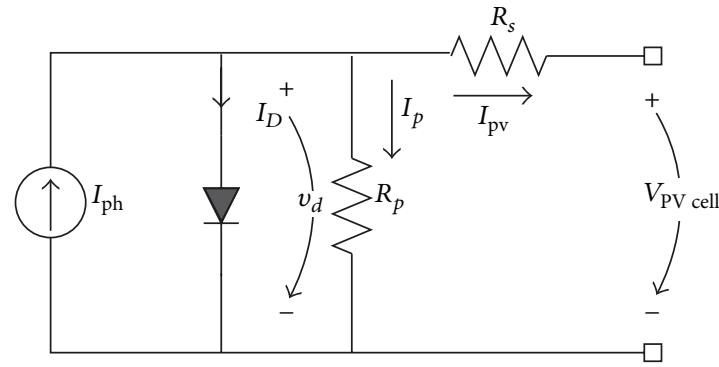

(a) Model based on a single-diode

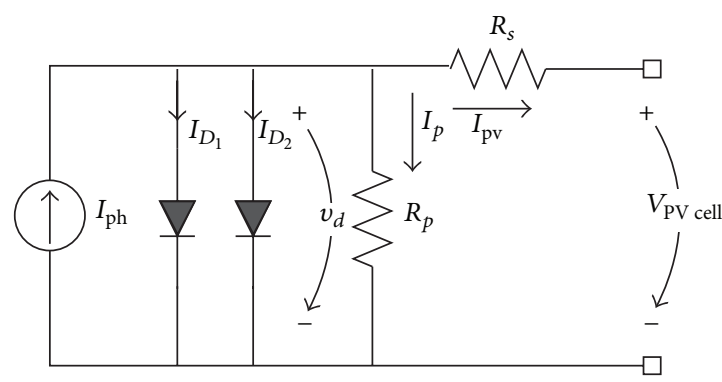

(b) Model based on a double-diode

FIgURE 2: Equivalent circuit of the PV cell.

TABLE 1: ZDNY-100C36 series PV module.

\begin{tabular}{lccc}
\hline \multirow{2}{*}{ Parameters } & \multicolumn{3}{c}{ Electrical parameters } \\
& Symbol & Value & Unit \\
\hline Maximum power & $P_{\max }$ & 100 & $\mathrm{~W}$ \\
Optimum operating voltage & $V_{m}$ & 17.5 & $\mathrm{~V}$ \\
Optimum operating current & $I_{m}$ & 5.71 & $\mathrm{~A}$ \\
Open-circuit voltage & $V_{\mathrm{oc}}$ & 21.98 & $\mathrm{~V}$ \\
Short-circuit current & $I_{\mathrm{sc}}$ & 6.16 & $\mathrm{~A}$ \\
Cell efficiency & $\eta_{c}$ & 17.30 & $\%$ \\
Module efficiency & $\eta_{m}$ & 14.50 & $\%$ \\
Number of cells in series & $N_{s}$ & 36 & - \\
\hline
\end{tabular}

applying Kirchoff's voltage law on the circuit of Figure 2(b) can be obtained by the following equation:

$$
V_{\mathrm{PV} \text { cell }}=v_{d}-R_{s} I_{\mathrm{pv}} \text {, }
$$

where $V_{\mathrm{PV} \text { cell }}$ is the voltage of the PV cell and $R_{s}$ is the equivalent series impedance. Considering the typical characteristics of the diode and combining the above equations, the output current is represented by the following equation:

$$
\begin{gathered}
I_{\mathrm{pv}}=I_{\mathrm{ph}}-I_{O}\left(e^{\xi\left(V_{\mathrm{PV} \text { cell }}+R_{s} I_{\mathrm{pv}}\right)}-1\right)-\frac{v_{d}}{R_{p}}, \\
\xi=\frac{q}{N_{s} \lambda k_{B} T},
\end{gathered}
$$

where $I_{O}$ is the reverse saturation current and the parameters $q, N_{s}, k_{B}, T$, and $\lambda$ are the electron charge $(q=1.6 \times$ $10^{-19} \mathrm{C}$ ), the number of series connected cells, Boltzmann's constant, the module temperature, and the ideal $p-n$ junction characteristic factor $(\lambda=1.3$, for monocrystalline solar cells), respectively.

2.1. Simulation Results. A Matlab/Simulink simulation environment is used for the analysis of the characteristics of a ZDNY-100C36 series PV module, and simulations have been performed to evaluate parametric simulations to analyze the MPPT for different irradiance conditions. Table 1 shows the typical electrical characteristics of the ZDNY100C36 module whose specifications are obtained under the standard test condition (STC) considering $1000 \mathrm{~W} / \mathrm{m}^{2}$ of irradiance, solar spectrum of air mass (AM) 1.5, and module temperature at $25^{\circ} \mathrm{C}$. In the parametric simulations different values of irradiation assuming a variation range between 200 and $1000 \mathrm{~W} / \mathrm{m}^{2}$ are considered. In one hand, Figure 3(a) shows the evolution of the current for different irradiation values, which in the case of the ZDNY-100C36 $\mathrm{PV}$ panel is equivalent to the optimum operating current $\left(I_{m}\right)$ that particularizing for the operating point equivalent to the STC can be quantified in $5.71 \mathrm{~A}$. On the other hand, Figure 3(b) shows the power evolution of the ZDNY-100C36 $\mathrm{PV}$ module assuming the same irradiance variation range. It can be seen that, considering the STC, the maximum power of the photovoltaic module is quantified at $100 \mathrm{~W}$. From this parametric simulations analysis it can be seen that a variation of $\pm 10 \%$ of the optimal operating point, caused due to a poor orientation of the PV module, may result in a deviation of $\pm 23.30 \%$ of the optimum power. In order to overcome these drawbacks the use of a novel design of a biaxial solar tracking system using ICTs is suggested.

\section{Biaxial Solar Tracking System Descriptions}

The proposed design has been modeled previously using mechanical design software, SolidWorks. The overall structural design of the biaxial solar tracking system is shown in Figure 4(a). The weight of the structure is approximately $800 \mathrm{~kg}$ and it has overall dimension of $7000 \mathrm{~mm} \times 5400 \mathrm{~mm} \times$ $1250 \mathrm{~mm}$. A side view and its dimension detail can be shown in Figure 4(b). The structure supports $24 \mathrm{PV}$ modules of the ZDNY-100C36 series. The main advantage of proposed topology is its lower resistance to the wind force compared with the conventional solution. It allows using lighter-weight dimensioning parts, which results in the benefit of the motors to be used in this application, especially when taking into account the power required to move the structure. To vary the elevation angle a linear actuator of the series SM4S900M3C3 is used, which is manufactured with stainless steel tubes and spindle and high carbon steel to be able to withstand a dynamic load of $8000 \mathrm{~N}$. The supply linear motor voltage is $24 \mathrm{~V} \mathrm{DC}$ and it allows a maximum current of $5 \mathrm{~A}$. The motor shaft is coupled to a high resolution rotary encoder E6JCWZ series, with a resolution of 600 pulses per revolution (ppr) used in this application to measure the displacement of the linear actuator that is related to the displacement of 


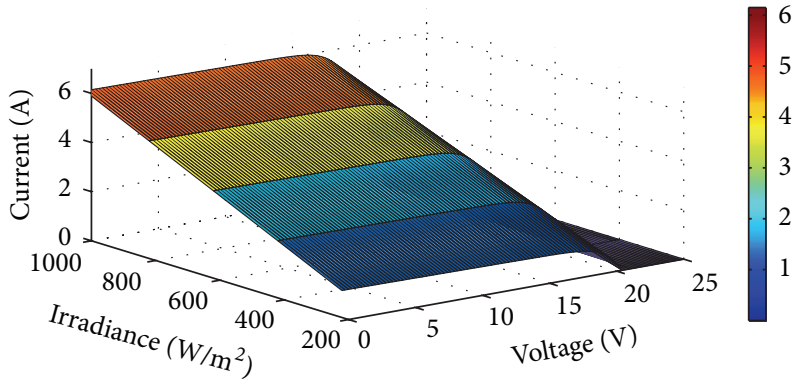

(a) Current versus voltage curves for different irradiation values

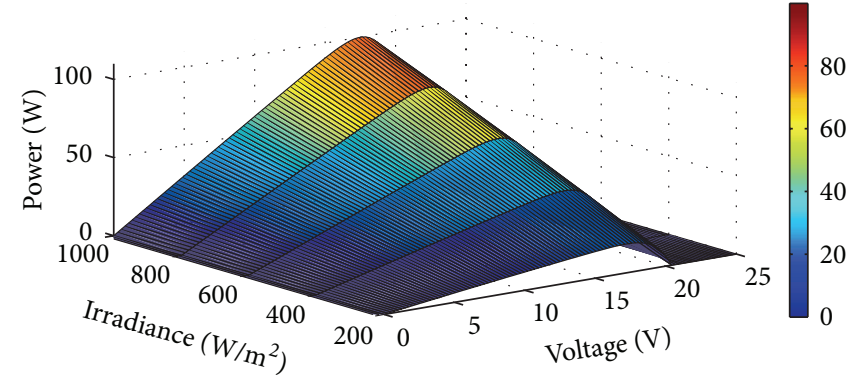

(b) Power versus voltage curves for different irradiation values

FIgURE 3: Parametric simulations of the ZDNY-100C36 series PV module.

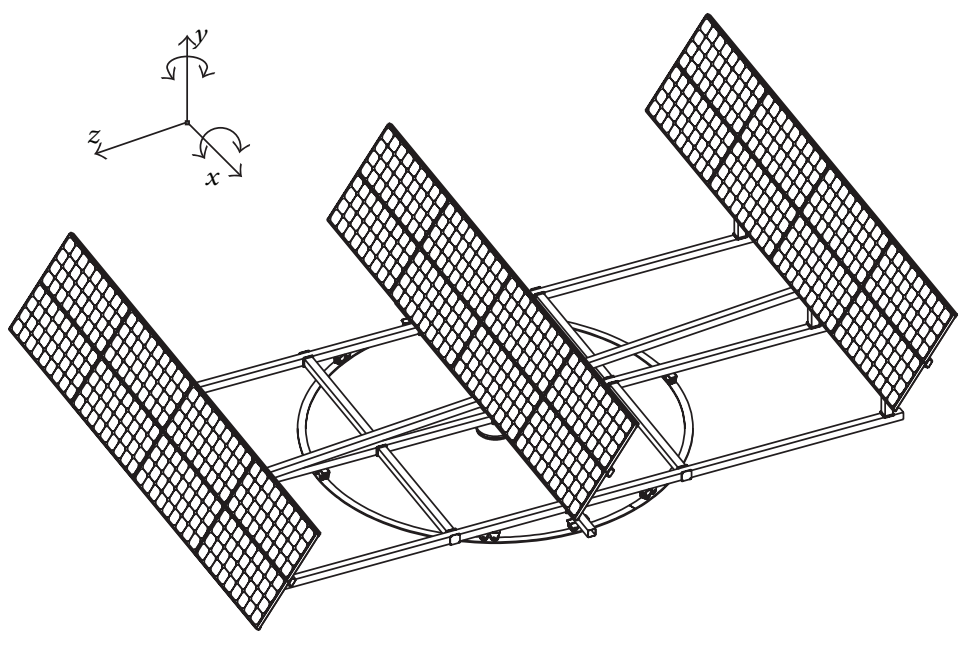

(a) Global design of the structure

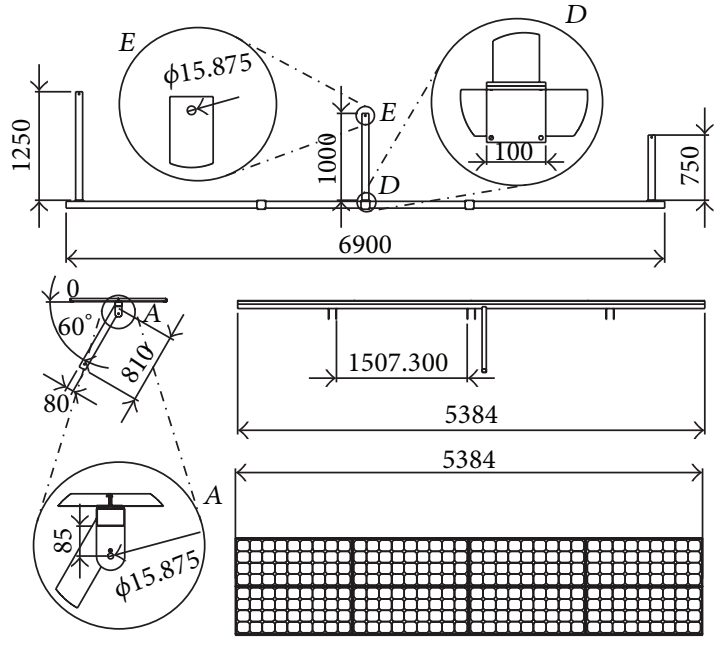

(b) Side view, dimensions, and details

FIGURE 4: Mechanical design based on the SolidWorks computational tool.

the structure in elevation angle. Then, to change the azimuth angle a $12 \mathrm{~V}$ DC motor is used and it coupled to a speed reduction system with a reduction ratio of $1: 70000$. The motor shaft is coupled to a high resolution incremental encoder (E6B2-CWZ6C series with a resolution of $400 \mathrm{ppr}$ ) to measure the position of the structure in azimuthal axis. In both cases, the position of the structure is determined in real-time and it is transmitted wirelessly using the 802.15.4 protocol to the main computer that is used for controlling and monitoring the PV plant. The reference for the control system (in azimuth and elevation angle) is provided by the P03/3-Modbus GPS weather station by using a proprietary algorithm. This device measures temperature, wind speed, and brightness (eastern, southern, and western sunlight). Moreover, it recognizes precipitation and it receives the Universal Time Coordinated (UTC) signals as well as the site coordinates via an integrated GPS receiver. The direction of the sun (azimuth) as well as its height (elevation) is calculated with the above details and then it is transmitted to the main computer wirelessly by a pair of series 2 XBee. Moreover, the references input to the control algorithm (azimuth and elevation angle) are transmitted using a pair of series 1 XBee
Pro. The initial and final positions of the biaxial solar tracking system are electronically detected by limit switches sensors. A detailed block diagram of the proposed biaxial solar tracking applied to the photovoltaic system is provided in Figure 1.

3.1. Elevation Mechanism. The different components used in the implementation of the biaxial solar tracking system for PV applications have been manufactured by using materials that comply with international standards of wind loads according to ISO 4354 specification. The overall system has been assembled according to the proposed design shown in Figure 4. On the other hand, Figure 5(a) showed the design of the elevation motion mechanism where the linear actuator coupled to an arm used to articulate the movement can be seen. Moreover, Figure 5(b) shows a picture of the elevation mechanism implemented.

3.1.1. Linear Motor Model. Assuming the generic transfer function (TF) of the DC motor as the relationship between the mechanical speed $(\omega)$ measured in revolutions per minute $(\mathrm{rpm})$ and the voltage applied to the motor $\left(V_{s e}\right)$ and considering the electrical and mechanical parameters, the motor 


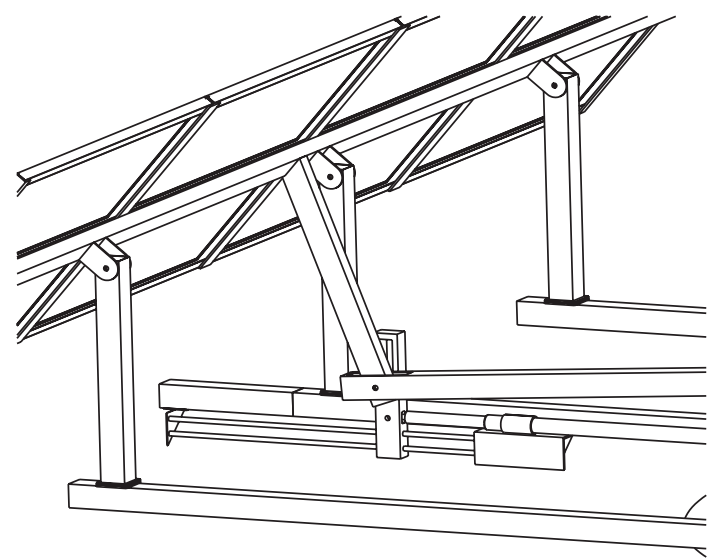

(a) Elevation mechanism design with SolidWorks computational tool

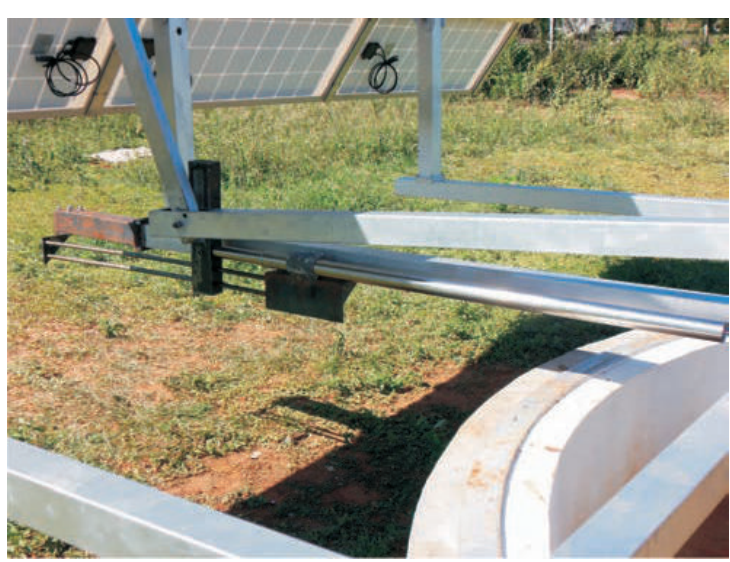

(b) Elevation mechanism implemented to articulate the movement

FIGURE 5: Mechanism of elevation motion and linear actuator.

model can be approximated by a second-order equation as follows:

$$
\frac{\omega(s)}{V_{s e}(s)}=\frac{K_{E e}}{L_{a e} J_{M e} s^{2}+\left(R_{a e} J_{M e}+D_{M e} L_{a e}\right) s+\left(K_{E e}^{2}+R_{a e} D_{M e}\right)},
$$

where $R_{a e}, L_{a e}, K_{E e}, J_{M e}$, and $D_{M e}$ are the armature electric resistance and the inductance, the back electromagnetic force constant, the moment of inertia, and the viscous friction coefficient, respectively. Table 2 shows the values of the parameters measured experimentally for the particular case of the linear actuator SM4S900M3C3. Considering a sampling frequency of $1.5 \mathrm{~Hz}$ and assuming that the TF is preceded by a zero-order hold ( $\mathrm{ZOH})$, the TF in discrete time can be written using the state-space representation approach as follows:

$$
\begin{gathered}
\mathbf{x}_{e(k+1 \mid k)}=\mathbf{A}_{e} \mathbf{x}_{e(k \mid k)}+\mathbf{B}_{e} \mathbf{u}_{e(k \mid k)}, \\
\mathbf{y}_{e(k \mid k)}=\mathbf{C}_{e} \mathbf{x}_{e(k \mid k)},
\end{gathered}
$$

where $\mathbf{u}_{e}$ is the input voltage, $\mathbf{x}_{e}=\left[\mathbf{V}_{s e}, \omega\right]^{T}$ is the state vector, and $\mathbf{A}_{e}$ and $\mathbf{B}_{e}$ are matrices that define the dynamics of the linear motor that for this set of state variables using the canonical form of control representation are defined as

$$
\begin{gathered}
\mathbf{A}_{e}=\left[\begin{array}{cc}
1.952314938166575 & -0.952378714704760 \\
1 & 0
\end{array}\right], \\
\mathbf{B}_{e}=\left[\begin{array}{ll}
1 & 0
\end{array}\right]^{T}, \\
\mathbf{C}_{e}=\left[\begin{array}{ll}
0.009456745359447 & 0.009304185583616
\end{array}\right] .
\end{gathered}
$$

Figure 6 shows the experimental validation of the mathematical model implemented using the Matlab/Simulink computational tool for 7 operation points in open loop. It can be seen in the figure that the step response obtained by simulations converges to the experimental values when different voltage excitation levels (from 12 to 24 volts, in step of $2 \mathrm{~V}$ ) are applied to the motor.
TABLE 2: Linear motor SM4S900M3C3 series parameters.

\begin{tabular}{lccc}
\hline \multirow{2}{*}{ Parameters } & \multicolumn{3}{c}{ Electrical and mechanical parameters } \\
& Symbol & Value & Unit \\
\hline Armature electric resistance & $R_{a e}$ & 1.425 & $\Omega$ \\
Armature electric inductance & $L_{a e}$ & 0.0015 & $\mathrm{H}$ \\
Back EMF constant & $K_{E e}$ & 0.031 & $\mathrm{~V} \cdot \mathrm{s} / \mathrm{rad}$ \\
Torque constant & $K_{T e}$ & 0.031 & $\mathrm{~V} \cdot \mathrm{s} / \mathrm{rad}$ \\
Current source & $i_{F e}$ & 0.27 & $\mathrm{~A}$ \\
Moment of inertia & $J_{M e}$ & $27 \times 10^{-6}$ & $\mathrm{~kg} \cdot \mathrm{m}^{2}$ \\
Viscous coefficient & $D_{M e}$ & $3.18 \times 10^{-6}$ & $\mathrm{Nm} \cdot \mathrm{s} / \mathrm{rad}$ \\
\hline
\end{tabular}

3.2. Azimuth Mechanism. Azimuth mechanism is based on a speed reduction system coupled to a DC motor, as shown in Figure 7(a). In order to reduce the speed of azimuth, the mechanism uses a combined system of two levels crownpinion using a chain drive coupled turn to the worm gear reducer box. The structure rotates in azimuthal direction using a ball bearing steel system to reduce the friction effects. This procedure allows the use of low power DC motors to move the PV system. The azimuth mechanism implemented is shown in Figure 7(b). The structure was manufactured from pieces formed by steel tubes and profiles using arc welding. The unions of the removable parts were performed using stainless steel screws. Each of the brackets, having $8 \mathrm{PV}$ modules of $100 \mathrm{~W}$ nominal power, has been arranged as shown in the photograph in Figure 1. The overall system designed provides a maximum power of $2.4 \mathrm{~kW}$.

3.2.1. Azimuthal Motor Model. In the same way as for the previous case electrical and mechanical parameters of azimuth motor have been measured experimentally in order to represent the mathematical model in the state-space. Table 3 shows the values of the parameters measured. In discrete time the mathematical model of the azimuth motor can be written as follows:

$$
\begin{gathered}
\mathbf{x}_{a(k+1 \mid k)}=\mathbf{A}_{a} \mathbf{x}_{a(k \mid k)}+\mathbf{B}_{a} \mathbf{u}_{a(k \mid k)}, \\
\mathbf{y}_{a(k \mid k)}=\mathbf{C}_{a} \mathbf{x}_{a(k \mid k)},
\end{gathered}
$$




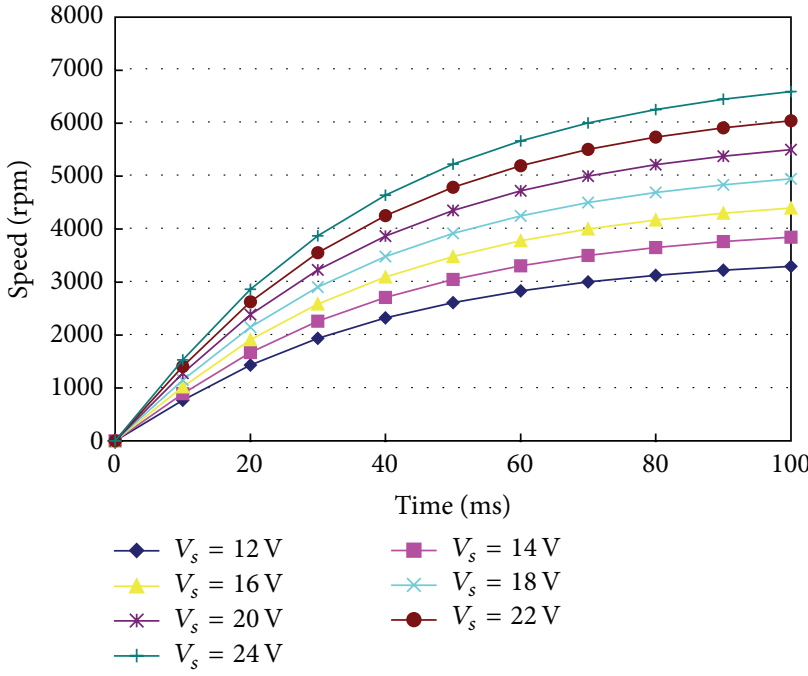

(a) Results obtained by simulations

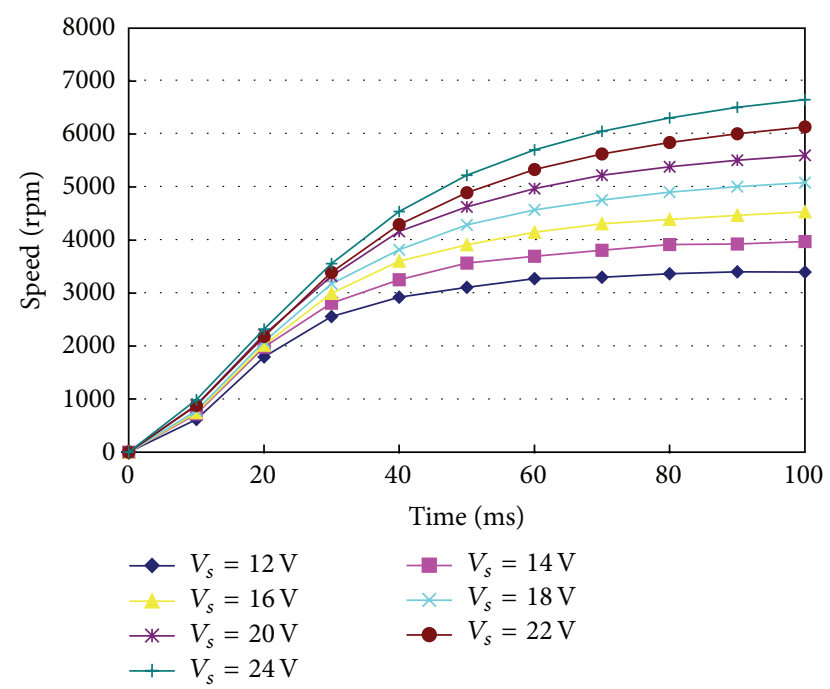

(b) Results obtained experimentally

FIGURE 6: Step response of the linear motor speed.

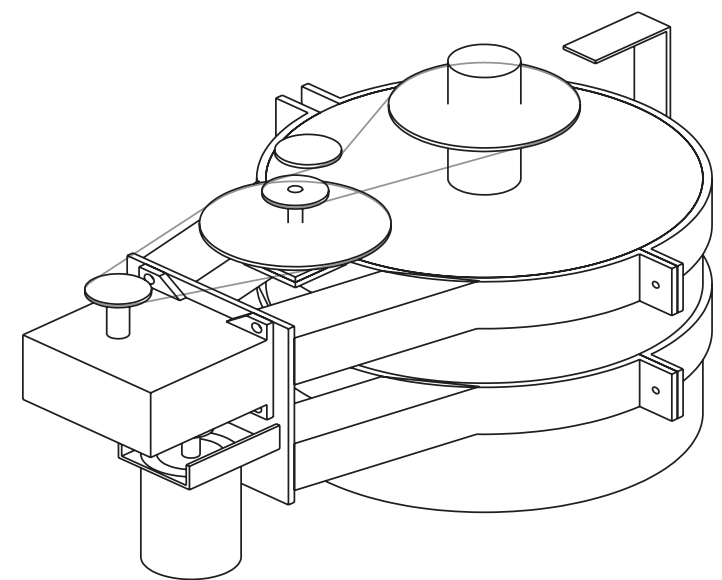

(a) Azimuth mechanism design with SolidWorks computational tool

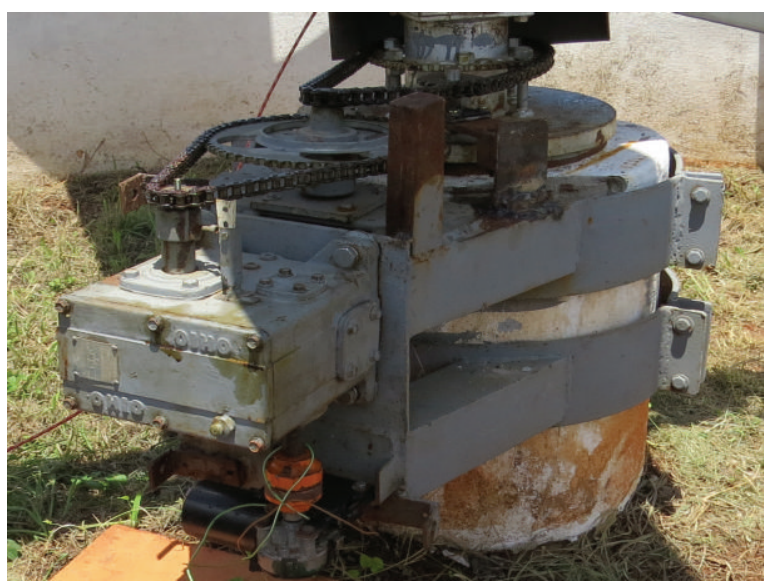

(b) Azimuth mechanism implemented to articulate the movement

FIGURE 7: Mechanism of azimuth motion and speed reduction system.

TABLE 3: Azimuth motor parameters.

\begin{tabular}{lccc}
\hline \multirow{2}{*}{ Parameters } & \multicolumn{3}{c}{ Electrical and mechanical parameters } \\
& Symbol & Value & Unit \\
\hline Armature electric resistance & $R_{a a}$ & 1.6 & $\Omega$ \\
Armature electric inductance & $L_{a a}$ & 0.002 & $\mathrm{H}$ \\
Back EMF constant & $K_{E a}$ & 0.0245 & $\mathrm{~V} \cdot \mathrm{s} / \mathrm{rad}$ \\
Torque constant & $K_{T a}$ & 0.0245 & $\mathrm{~V} \cdot \mathrm{s} / \mathrm{rad}$ \\
Current source & $i_{F a}$ & 0.754 & $\mathrm{~A}$ \\
Moment of inertia & $J_{M a}$ & $16 \times 10^{-6}$ & $\mathrm{~kg} \cdot \mathrm{m}^{2}$ \\
Viscous coefficient & $D_{M a}$ & $16 \times 10^{-6}$ & $\mathrm{Nm} \cdot \mathrm{s} / \mathrm{rad}$ \\
\hline
\end{tabular}

where $\mathbf{u}_{a}$ is the input voltage, $\mathbf{x}_{a}=\left[\mathbf{V}_{s a}, \omega_{a}\right]^{T}$ is the state vector, and $\mathbf{A}_{a}$ and $\mathbf{B}_{a}$ are matrices that define the dynamics of the azimuthal motor that for this set of state variables using are defined as

$$
\begin{aligned}
& \mathbf{A}_{a}=\left[\begin{array}{cc}
1.959704907964181 & -0.959755299777461 \\
1 & 0
\end{array}\right], \\
& \mathbf{B}_{a}=\left[\begin{array}{ll}
1 & 0
\end{array}\right]^{T}, \\
& \mathrm{C}_{a}=1 \times 10^{-4}[0.758665307052543 \quad 0.748348332271132] \text {. }
\end{aligned}
$$

Figure 8 shows the experimental validation of the mathematical model implemented using the Matlab/Simulink computational tool for 4 operation points in open loop. It can be seen in the figure that the step response obtained by simulations converges to the experimental values when it is applied to the motor different voltage excitation levels, from 8 to 14 volts in step of $2 \mathrm{~V}$, respectively. 


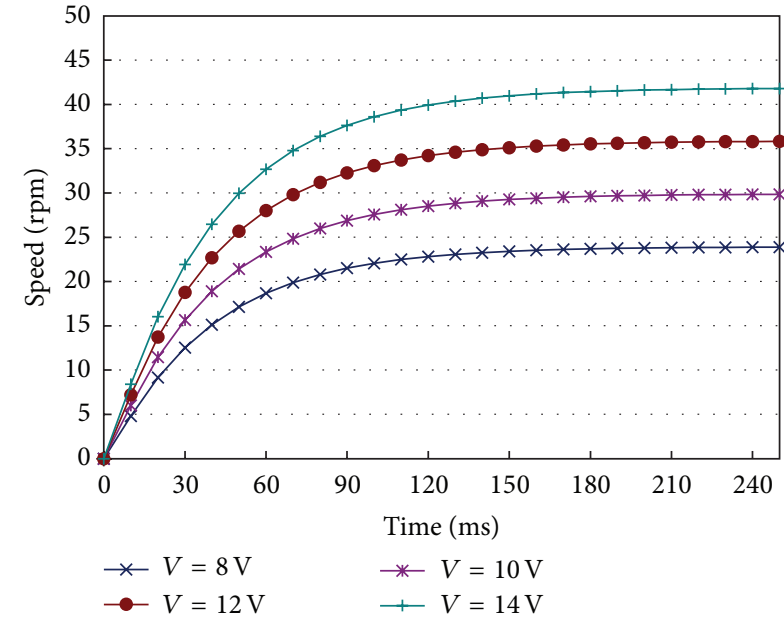

(a) Results obtained by simulations



(b) Results obtained experimentally

FIGURE 8: Step response of the azimuth motor speed.

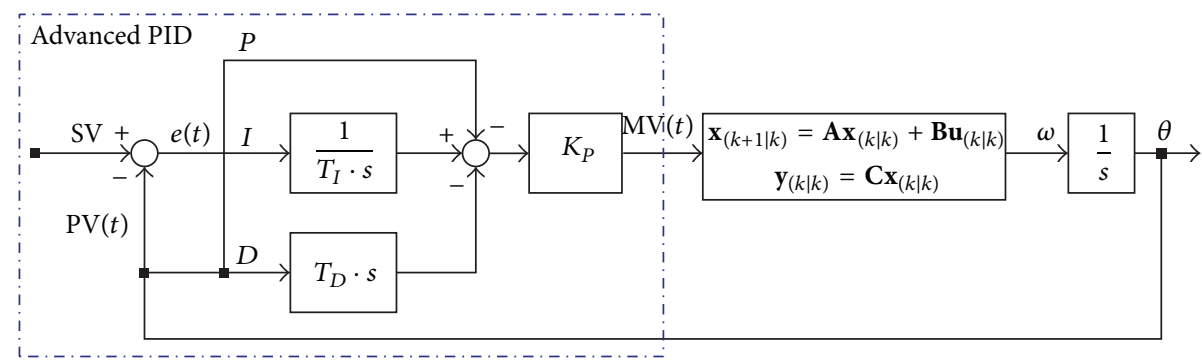

FIGURE 9: Advanced-PID position controller.

\section{Control Strategy}

In order to control the position of the structure, an advancedproportional integral derivative (Advanced-PID) control algorithm has been implemented. The main advantage of the Advanced-PID control approach is its low computational burden to carry out the control. The performance of the Advanced-PID can be also modified by varying the three control variables. In the proposed scheme the Advanced-PID controller computes an error value as the difference between a measured variable (the structure position obtained by the encoder sensors) and a desired setpoint (the sun position obtained from the GPS by the weather station). The controller attempts to minimize the error by adjusting the process control inputs using the scheme shown in the block diagram of Figure 9.

The discrete implementation can be obtained from the block diagram using approximations for first order to derivatives by backward finite differences [16]. Thus the integral and derivative terms can be discretized with a sampling time $\Delta t$; as a result the control equations can be written as follows:

$$
\Delta M V_{k}=K_{P}\left(\left(e_{k}-e_{k-1}\right)+\frac{\Delta t}{T_{I}} e_{k}+\frac{T_{D}}{\Delta t}\left(e_{k}-2 e_{k-1}+e_{k-2}\right)\right)
$$

$$
M V_{k}=M V_{k-1}+\Delta M V_{k}
$$

where $K_{P}, T_{I}, T_{D}$, and $e_{k}$ represent the proportional gain, integral and derivative time, and the error, respectively. In order to minimize the effects of the noise on the proposed control strategy, the term $\left(e_{k}-e_{k-1}\right)$ in (9) is replaced by $\left(\mathrm{PV}_{k-1}-\mathrm{PV}_{k}\right)$. Algorithm 1 shows the pseudocode AdvancedPID control algorithm, where the control effort is applied to the azimuth and elevation mechanisms at each sampling time in terms of duty cycle.

An interval control mode for small displacements has been applied to the simultaneous (elevation and azimuth) motors in order to evaluate the efficiency of the AdvancedPID control algorithm. The result of the interval control is shown in Figure 10. Advanced-PID position control was performed under a PWM frequency of $19.5 \mathrm{kHz}$ and the values of proportional gain and integral and derivative time $\left(K_{P}=20\right.$, $T_{I}=0.6$, and $T_{D}=0.3$ ) have been calculated initially using the Ziegler-Nichols method and have been subsequently adjusted by heuristic rules to obtain an acceptable dynamic response. Figure 10(a) shows the multistep response of the linear motor for small displacements. This figure shows that the proposed Advanced-PID position control implemented has a good dynamic response considering parameters in the time-domain tests as rise time (around $3 \mathrm{~s}$ ), settling time (around $5 \mathrm{~s}$ ), and overshoot and steady-state error (near zero). 


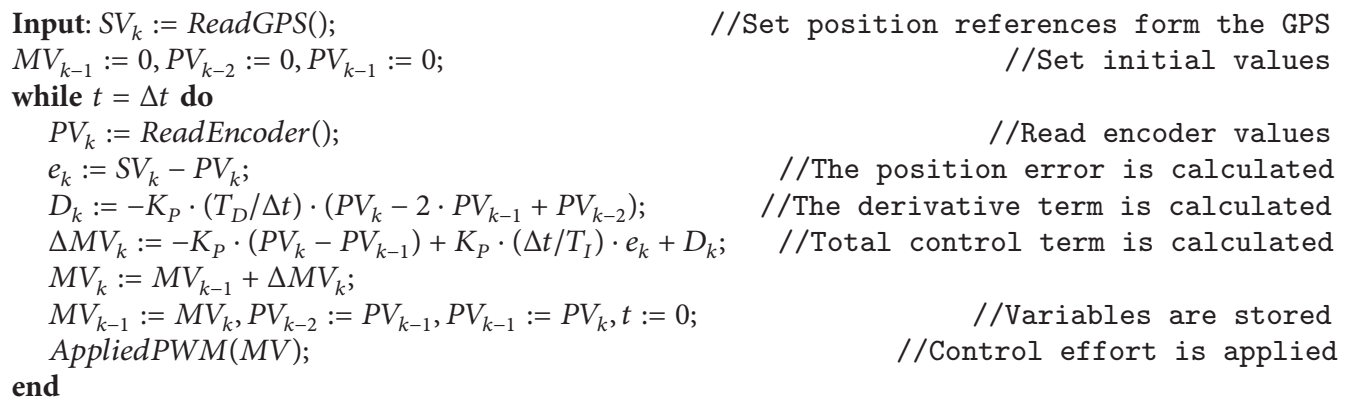

//Set position references form the GPS

//Set initial values

//Read encoder values

//The position error is calculated

//The derivative term is calculated

//Total control term is calculated

//Variables are stored

//Control effort is applied

Algorithm 1: Advanced-PID position controller.

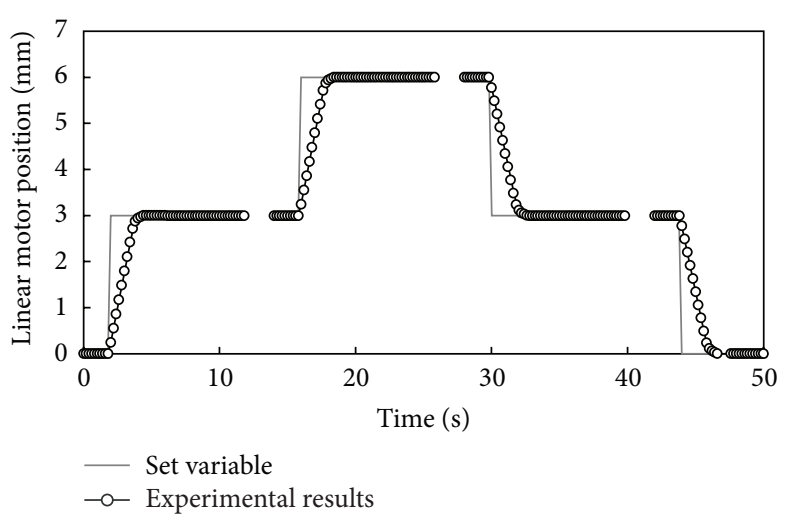

(a) Linear motor response for $K_{P}=20, T_{I}=0.6$, and $T_{D}=0.3$

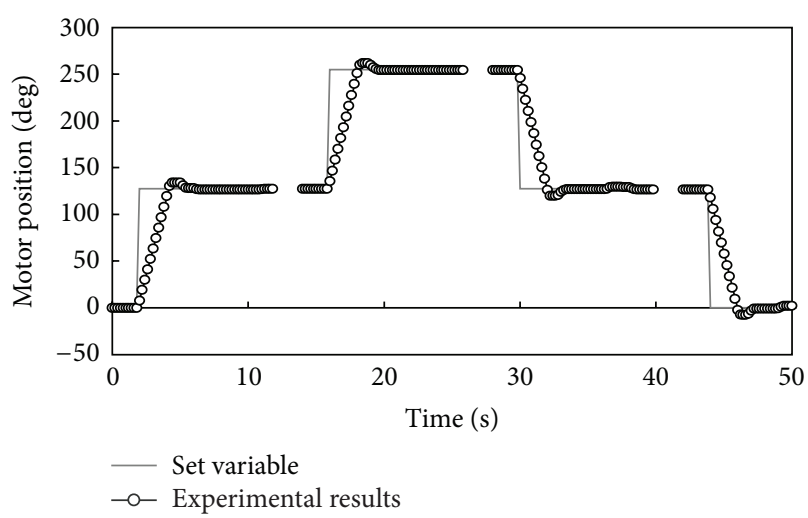

(b) Azimuthal motor response for $K_{P}=20, T_{I}=0.6$, and $T_{D}=0.3$

FIgURE 10: Position step response of the linear and azimuth motors for small displacements.

Moreover, Figure 10(b) shows the multistep angle response of the azimuth motor, where it can be seen that the dynamic response converges to the reference values with steady-state error (near zero).

Figure 11 shows the experimental results for medium displacements and for different values of proportional gain and integral and derivative time $\left(K_{P}=20, T_{I}=1.0\right.$, and $T_{D}=$ 0.6 ). As can be seen in Figure 11(a) (for the case of the lineal motor) and Figure 11(b) (for the case of the azimuth motor) the multistep response converges to the reference values with steady-state error (near zero), even if it is considered medium displacements.

\section{Experimental Results}

The proposed control strategy was tested in the $2.4 \mathrm{~kW}$ biaxial tracking system, taking a variable elevation and azimuth sun's angle as the reference, provided by the P03/3-Modbus GPS weather station as shown in Figure 12(a). The linear and azimuth motors were fed through an H-Bridge driver used to generate two independent $19.5 \mathrm{kHz}$ PWM signals based on the locked antiphase (LA) technique. The Advanced-PID position control was performed under a sampling frequency of $1.5 \mathrm{~Hz}$ using the following values of gains for the PID algorithm: $K_{P}=20, T_{I}=0.6$, and $T_{D}=0.3$, which have been tuned by heuristic rules to obtain an acceptable dynamic response. Figure 12(b) shows a comparative analysis of the efficiency between the proposed biaxial tracking system taking as reference the fixed system, under the same electrical connection scheme and load conditions. The total power generated was quantified in 5-minute intervals under noideal weather conditions (slightly cloudy) for both topologies (biaxial and fixed system). The fixed system was positioned with an elevation angle of 25.2972 degrees (which is the same as the site's latitude where the biaxial solar tracking system was implemented) and the azimuth was zero degrees to get it oriented in north-south direction. As shown in Figure 12(b), the biaxial solar tracking system yielded higher power and lower degradation of the power than the fixed system within $1 \mathrm{~h}$ of testing. The results show that the proposed biaxial solar tracking system introduces an average improvement of $37 \%$ compared with the results obtained from a fixed solar system.

\section{Conclusion}

The field of renewable energies has experienced a substantial growth in the last decades in which the photovoltaic application has been one of the most active research areas due to the costs reduction and increment of efficiency. Research has 


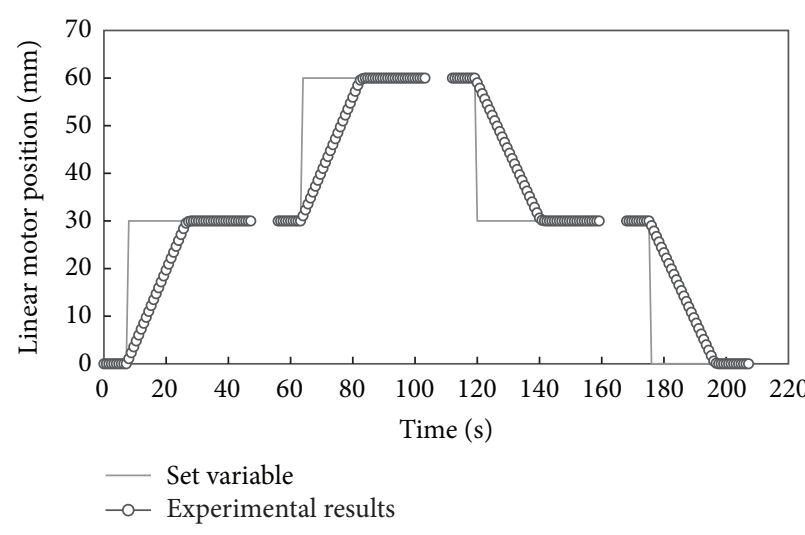

(a) Linear motor response for $K_{P}=20, T_{I}=1.0$, and $T_{D}=0.6$

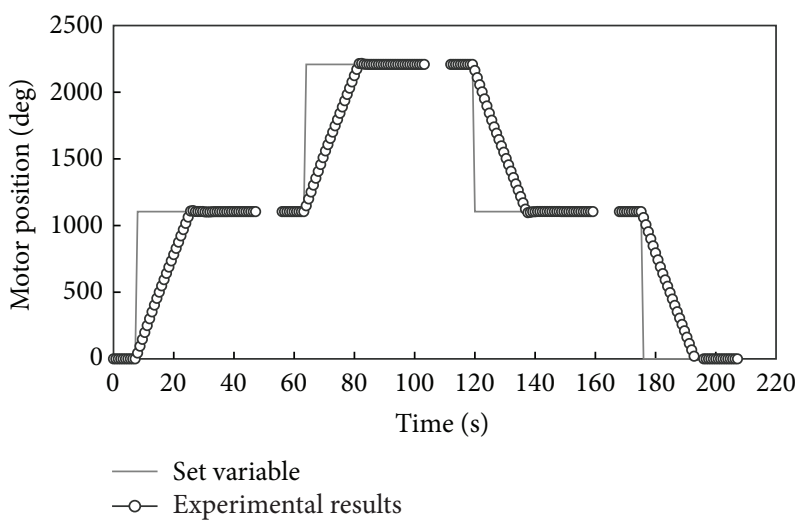

(b) Azimuthal motor response for $K_{P}=20, T_{I}=1.0$, and $T_{D}=0.6$

FIGURE 11: Position step response of the linear and azimuth motors for medium displacements.

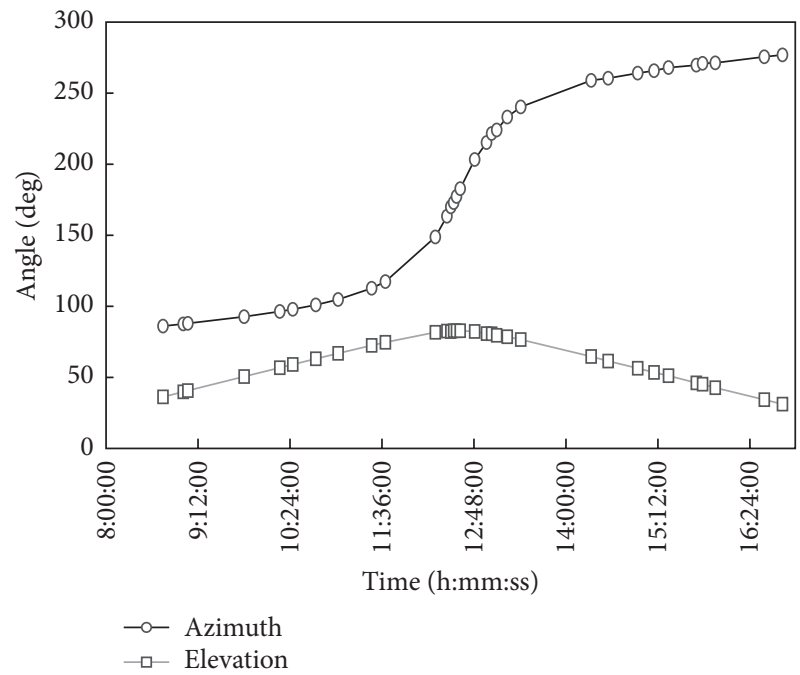

(a) Azimuth and elevation of the sun measured by the weather station on November 27, 2014

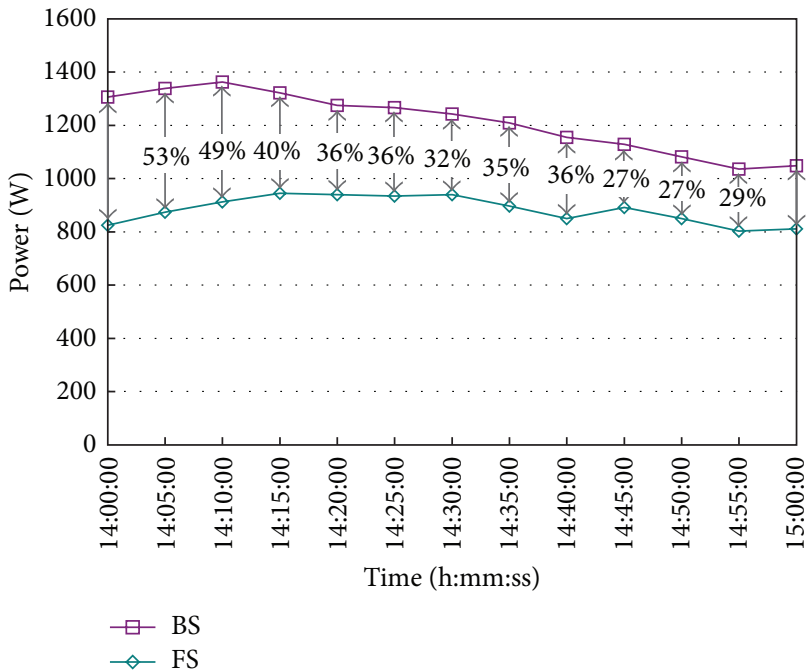

(b) Comparative analysis of efficiency between the biaxial (BS) and fixed system (FS)

Figure 12: Experimental results measured on November 27, 2014.

been recently conducted worldwide and numerous interesting developments have been reported in the literature from several complementary aspects to maximize the efficiency and extract the maximum power from the PV systems. In this paper a novel design of a biaxial solar tracking system has been proposed using ICTs to improve the efficiency of the photovoltaic system implementing the Advanced-PID control of the structure in order to achieve the optimal position of the PV modules perpendicular to the sun rays in real-time. Finally, experimental results have been measured in order to evaluate the proposed system and control performance with respect to the fixed solar system. The comparative analysis of efficiency shows that the performance in terms of generated power from the biaxial solar tracking system can be quantified (in average) in the order of $37 \%$, showing a tendency to increase when a drastic azimuth sun change occurs.

\section{Conflict of Interests}

The authors declare that there is no conflict of interests regarding the publication of this paper.

\section{Acknowledgments}

The authors would like to thank the Paraguayan Government for the economic support provided by means of a CONACYT grant (Project 10INV13). In addition, they wish to express their gratitude to the anonymous reviewers for their helpful comments and suggestions.

\section{References}

[1] A. Al-Diab and C. Sourkounis, "Variable step size P\&O MPPT algorithm for PV systems," in Proceedings of the 12th International Conference on Optimization of Electrical and Electronic 
Equipment (OPTIM '10), pp. 1097-1102, IEEE, Braşov, Romania, May 2010.

[2] R. S. Munoz-Aguilar, P. Rodriguez, G. Vazquez, I. Candela, and E. Aldabas, "Efficiency analysis of DCM-232 three-phase PV topology," in Proceedings of the 38th Annual Conference on IEEE Industrial Electronics Society (IECON '12), pp. 5714-5719, Montreal, Canada, October 2012.

[3] J. Shi, W.-J. Lee, Y. Liu, Y. Yang, and P. Wang, "Forecasting power output of photovoltaic systems based on weather classification and support vector machines," IEEE Transactions on Industry Applications, vol. 48, no. 3, pp. 1064-1069, 2012.

[4] B. Subudhi and R. Pradhan, "A comparative study on maximum power point tracking techniques for photovoltaic power systems," IEEE Transactions on Sustainable Energy, vol. 4, no. 1, pp. 89-98, 2013.

[5] A. Al Nabulsi and R. Dhaouadi, "Efficiency optimization of a dsp-based standalone PV system using fuzzy logic and dualMPPT control," IEEE Transactions on Industrial Informatics, vol. 8, no. 3, pp. 573-584, 2012.

[6] H. Mousazadeh, A. Keyhani, A. Javadi, H. Mobli, K. Abrinia, and A. Sharif, "A review of principle and sun-tracking methods for maximizing solar systems output," Renewable and Sustainable Energy Reviews, vol. 13, no. 8, pp. 1800-1818, 2009.

[7] M. J. Clifford and D. Eastwood, "Design of a novel passive solar tracker," Solar Energy, vol. 77, no. 3, pp. 269-280, 2004.

[8] F. R. Rubio, M. G. Ortega, F. Gordillo, and M. Lopez-Martinez, "Application of new control strategy for sun tracking," Energy Conversion and Management, vol. 48, no. 7, pp. 2174-2218, 2007.

[9] C. S. Chin, A. Babu, and W. McBride, "Design, modeling and testing of a standalone single axis active solar tracker using MATLAB/Simulink," Renewable Energy, vol. 36, no. 11, pp. 3075-3090, 2011.

[10] F. Scarpetta, M. Liserre, and R. A. Mastromauro, "Adaptive distributed MPPT algorithm for photovoltaic systems," in Proceedings of the 38th Annual Conference on IEEE Industrial Electronics Society (IECON '12), pp. 5708-5713, Montreal, Canada, October 2012.

[11] K. Ding, X. Bian, H. Liu, and T. Peng, "A MATLAB-simulinkbased PV module model and its application under conditions of nonuniform irradiance," IEEE Transactions on Energy Conversion, vol. 27, no. 4, pp. 864-872, 2012.

[12] W. Xiao, F. F. Edwin, G. Spagnuolo, and J. Jatskevich, "Efficient approaches for modeling and simulating photovoltaic power systems," IEEE Journal of Photovoltaics, vol. 3, no. 1, pp. 500$508,2013$.

[13] L. V. Hartmann, M. A. Vitorino, M. B. Rossite, and A. M. N. Lima, "Combining model-based and heuristic techniques for fast tracking the maximum-power point of photovoltaic systems," IEEE Transactions on Power Electronics, vol. 28, no. 6, pp. 2875-2885, 2013.

[14] D. Giaffreda, P. Magnone, M. Meneghini et al., "Local shunting in multicrystalline silicon solar cells: distributed electrical simulations and experiments," IEEE Journal of Photovoltaics, vol. 4, no. 1, pp. 40-47, 2014.

[15] Y. A. Mahmoud, W. Xiao, and H. H. Zeineldin, "A parameterization approach for enhancing PV model accuracy," IEEE Transactions on Industrial Electronics, vol. 60, no. 12, pp. 57085716, 2013.

[16] G. Franklin, J. D. Powell, and M. L. Workman, "Review of continuous control," in Digital Control of Dynamic Systems, pp. 23-24, Addison-Wesley, Menlo Park, Calif, USA, 3rd edition, 1998. 

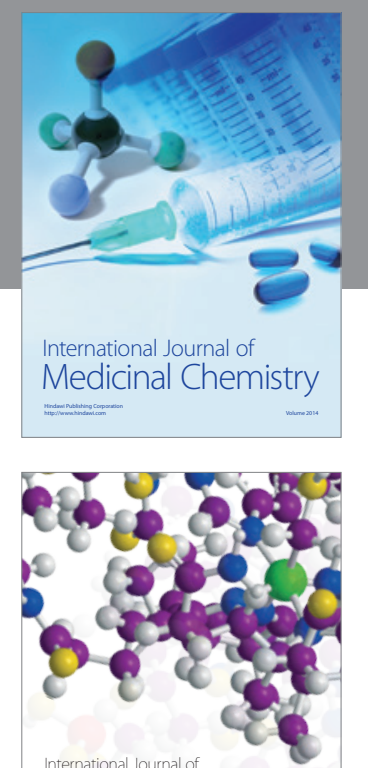

\section{Carbohydrate} Chemistry

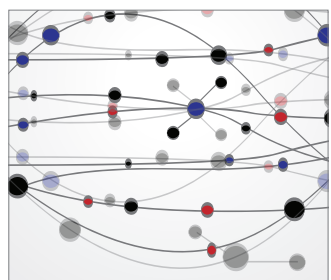

The Scientific World Journal
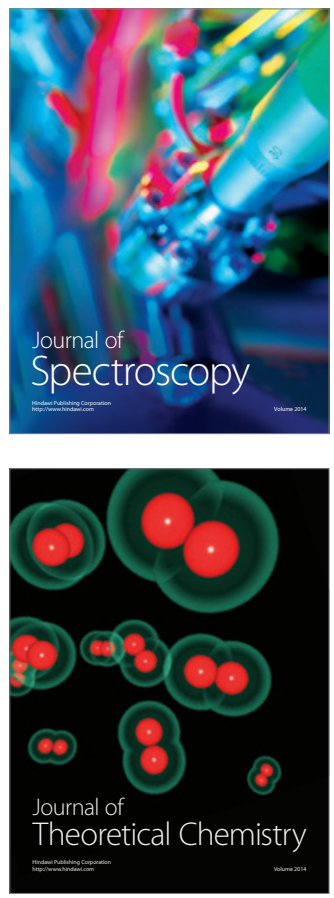
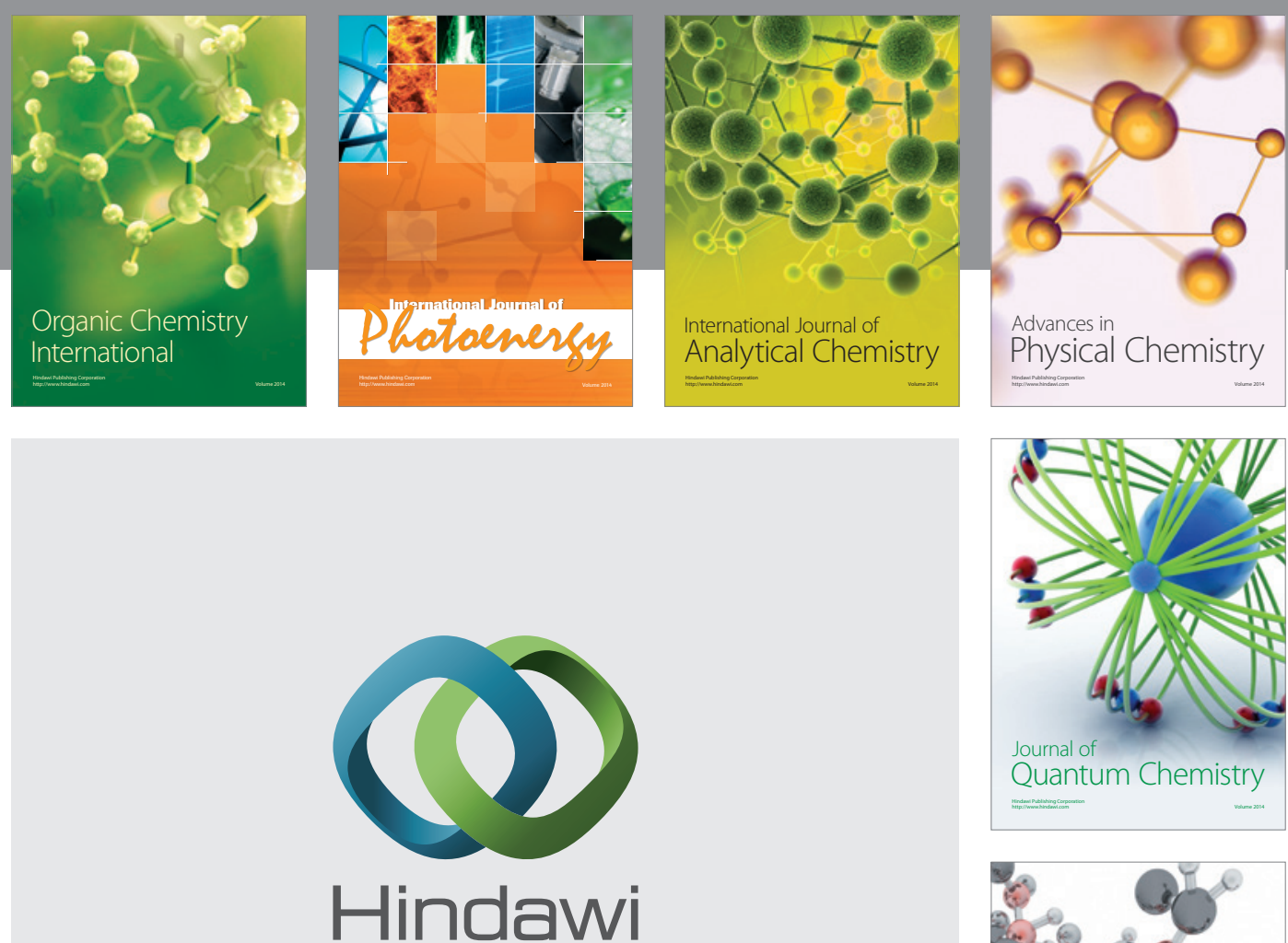

Submit your manuscripts at

http://www.hindawi.com

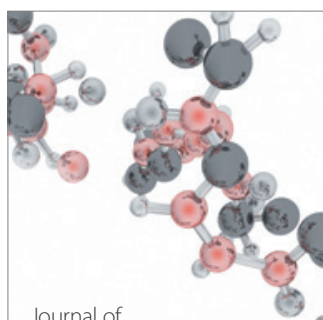

Analytical Methods

in Chemistry

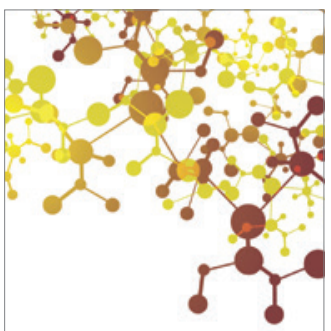

Journal of

Applied Chemistry

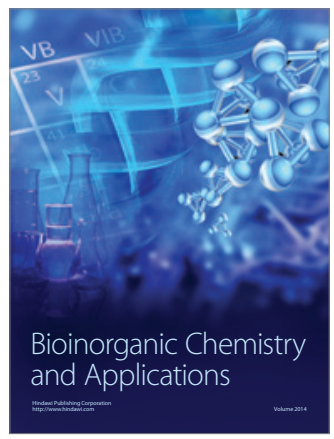

Inorganic Chemistry
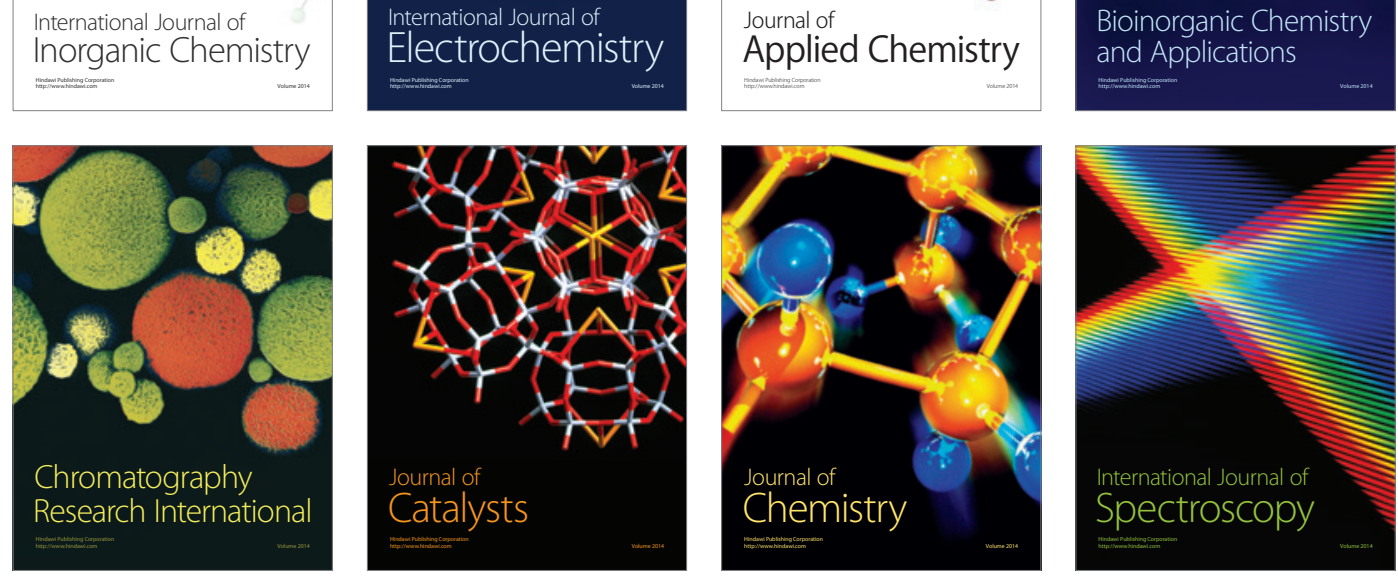